\title{
Nonlinear singular problems with indefinite potential term
}

\author{
Nikolaos S. Papageorgiou ${ }^{1,2} \cdot$ Vicenţiu D. Rădulescu2,3,4 D Dušan D. Repovš ${ }^{2,5}$
}

Received: 16 May 2019 / Revised: 16 May 2019 / Accepted: 15 June 2019 / Published online: 24 June 2019

(c) The Author(s) 2019

\begin{abstract}
We consider a nonlinear Dirichlet problem driven by a nonhomogeneous differential operator plus an indefinite potential. In the reaction we have the competing effects of a singular term and of concave and convex nonlinearities. In this paper the concave term will be parametric. We prove a bifurcation-type theorem describing the changes in the set of positive solutions as the positive parameter $\lambda$ varies.
\end{abstract}

Keywords Nonhomogeneous differential operator · Indefinite potential · Singular term · Concave and convex nonlinearities · Truncation · Comparison principles · Nonlinear regularity $\cdot$ Nonlinear maximum principle

Mathematics Subject Classification $35 \mathrm{~J} 75 \cdot 35 \mathrm{~J} 92 \cdot 35 \mathrm{P} 30$

\ Vicenţiu D. Rădulescu vicentiu.radulescu@imar.ro

Nikolaos S. Papageorgiou npapg@math.ntua.gr

Dušan D. Repovš dusan.repovs@guest.arnes.si

1 Department of Mathematics, Zografou Campus, National Technical University, 15780 Athens, Greece

2 Institute of Mathematics, Physics and Mechanics, 1000 Ljubljana, Slovenia

3 Faculty of Applied Mathematics, AGH University of Science and Technology, 30-059 Kraków, Poland

4 Department of Mathematics, University of Craiova, 200585 Craiova, Romania

5 Faculty of Education and Faculty of Mathematics and Physics, University of Ljubljana, 1000 Ljubljana, Slovenia 


\section{Introduction}

Let $\Omega \subseteq \mathbb{R}^{N}$ be a bounded domain with a $C^{2}$-boundary $\partial \Omega$. In this paper we study the following nonlinear nonhomogeneous parametric singular problem:

$$
\left\{\begin{array}{l}
-\operatorname{div} a(D u(z))+\xi(z) u(z)^{p-1}=\vartheta(u(z))+\lambda u(z)^{q-1}+f(z, u(z)) \text { in } \Omega \\
\left.u\right|_{\partial \Omega}=0, u>0, \lambda>0,1<q<p<\infty
\end{array}\right\}
$$

The map $a: \mathbb{R}^{N} \rightarrow \mathbb{R}^{N}$ involved in the differential operator of $\left(P_{\lambda}\right)$ is strictly monotone, continuous (hence maximal monotone, too) and satisfies certain other regularity and growth conditions which are listed in hypotheses $H(a)$ below (see Sect. 2). These conditions are general enough to incorporate in our framework many differential operators of interest such as the $p$-Laplacian and the $(p, q)$-Laplacian (that is, the sum of a $p$-Laplacian and a $q$-Laplacian). The operator $u \mapsto \operatorname{div} a(D u)$ is not homogeneous and this is a source of difficulties in the analysis of problem $\left(P_{\lambda}\right)$. The potential function $\xi \in L^{\infty}(\Omega)$ is indefinite (that is, sign changing). So the operator $u \mapsto-\operatorname{div} a(D u)+\xi(z)|u|^{p-2} u$ is not coercive and this is one more difficulty in the analysis of problem $\left(P_{\lambda}\right)$. In the reaction (the right-hand side of $\left(P_{\lambda}\right)$ ), the term $\vartheta(\cdot)$ is singular at $x=0$, while the perturbation contains the combined effects of a parametric concave term $x \mapsto \lambda x^{q-1}(x \geqslant 0)$ (recall that $q<p$ ), with $\lambda>0$ being the parameter and of a Carathéodory function $f(z, x)$ (that is, for all $x \in \mathbb{R}$ the mapping $z \mapsto f(z, x)$ is measurable and for almost all $z \in \Omega$ the mapping $x \mapsto f(z, x)$ is continuous), which is assumed to exhibit ( $p-1)$-superlinear growth near $+\infty$, but without satisfying the usual for superlinear problems Ambrosetti-Rabinowitz condition (the AR-condition for short). So in problem $\left(P_{\lambda}\right)$ we have the competing effects of singular, concave and convex terms.

Using variational methods related to the critical point theory, combined with suitable truncation, perturbation and comparison techniques, we produce a critical parameter value $\lambda^{*}>0$ such that

(i) for all $\lambda \in\left(0, \lambda^{*}\right)$ problem $\left(P_{\lambda}\right)$ has at least two positive solutions;

(ii) for $\lambda=\lambda^{*}$ problem $\left(P_{\lambda}\right)$ has at least one positive solution;

(iii) for all $\lambda>\lambda^{*}$ problem $\left(P_{\lambda}\right)$ has no positive solutions.

This work continues the recent paper by Papageorgiou et al. [16], where $\xi \equiv 0$ and in the reaction the parametric term is the singular one. It is also related to the works of Papageorgiou and Smyrlis [17] and Papageorgiou and Winkert [19], where the differential operator is the $p$-Laplacian, $\xi \equiv 0$ and no concave terms are allowed. Singular $p$-Laplacian equations with no potential term and reactions of special form were considered by Chu et al. [2], Giacomoni et al. [5], Li and Gao [10], Mohammed [12], Perera and Zhang [20], and Papageorgiou et al. [14]. 


\section{Mathematical background and hypotheses}

In this section we present the main mathematical tools which we will use in the analysis of problem $\left(P_{\lambda}\right)$. We also fix our notation and state the hypotheses on the data of the problem.

So, let $X$ be a Banach space, $X^{*}$ its topological dual, and let $\varphi \in C^{1}(X)$. We say that $\varphi(\cdot)$ satisfies the "C-condition", if the following property holds:

"Every sequence $\left\{u_{n}\right\}_{n} \geqslant 1 \subseteq X$ such that $\left\{\varphi\left(u_{n}\right)\right\}_{n \geqslant 1} \subseteq \mathbb{R}$ is bounded and $\left(1+\left\|u_{n}\right\|_{X}\right) \varphi^{\prime}\left(u_{n}\right) \rightarrow 0$ in $X^{*}$ as $n \rightarrow \infty$, admits a strongly convergent subsequence".

This is a compactness-type condition on the functional $\varphi(\cdot)$, which leads to the minimax theory of the critical values of $\varphi(\cdot)$ (see, for example, Papageorgiou et al. [15]). We denote by $K_{\varphi}$ the critical set of $\varphi$, that is,

$$
K_{\varphi}=\left\{u \in X: \varphi^{\prime}(u)=0\right\} .
$$

The main spaces in the analysis of problem $\left(P_{\lambda}\right)$ are the Sobolev space $W_{0}^{1, p}(\Omega)$ $(1<p<\infty)$ and the Banach space $C_{0}^{1}(\bar{\Omega})=\left\{u \in C^{1}(\bar{\Omega}):\left.u\right|_{\partial \Omega}=0\right\}$. We denote by $\|\cdot\|$ the norm of $W_{0}^{1, p}$. By the Poincaré inequality we have

$$
\|u\|=\|D u\|_{p} \text { for all } u \in W_{0}^{1, p}(\Omega)
$$

The Banach space $C_{0}^{1}(\Omega)$ is ordered with positive (order) cone

$$
C_{+}=\left\{u \in C_{0}^{1}(\bar{\Omega}): u(z) \geqslant 0 \text { for all } z \in \bar{\Omega}\right\} .
$$

This cone has a nonempty interior given by

$$
\text { int } C_{+}=\left\{u \in C_{+}: u(z)>0 \text { for all } z \in \Omega,\left.\frac{\partial u}{\partial n}\right|_{\partial \Omega}<0\right\} \text {, }
$$

with $n(\cdot)$ being the outward unit normal on $\partial \Omega$.

We will also use two additional ordered Banach spaces. The first one is

$$
C_{0}(\bar{\Omega})=\left\{u \in C(\bar{\Omega}):\left.u\right|_{\partial \Omega}=0\right\}
$$

This cone is ordered with positive (order) cone

$$
K_{+}=\left\{u \in C_{0}(\bar{\Omega}): u(z) \geqslant 0 \text { for all } z \in \bar{\Omega}\right\}
$$

This cone has a nonempty interior given by

$$
\text { int } K_{+}=\left\{u \in K_{+}: c_{u} \hat{d} \leqslant u \text { for some } c_{u}>0\right\} \text {, }
$$


where $\hat{d}(z)=d(z, \partial \Omega)$ for all $z \in \bar{\Omega}$. On account of Lemma 14.16 of Gilbarg and Trudinger [6, p. 355], we have

$$
\text { " } c_{u} \hat{d} \leqslant u \text { for some } c_{u}>0 \text { if and only if } \hat{c}_{u} \hat{u}_{1} \leqslant u \text { for some } \hat{c}_{u}>0 \text { ", }
$$

with $\hat{u}_{1}$ being the positive, $L^{p}$-normalized (that is, $\left\|\hat{u}_{1}\right\|_{p}=1$ ) eigenfunction corresponding to the principal eigenvalue $\hat{\lambda}_{1}>0$ of the Dirichlet $p$-Laplacian. The nonlinear regularity theory and the nonlinear maximum principle (see, for example, Gasinski and Papageorgiou [4, pp. 737-738]), imply that $\hat{u}_{1} \in \operatorname{int} C_{+}$.

The second ordered space is $C^{1}(\bar{\Omega})$ with positive (order) cone

$$
\hat{C}_{+}=\left\{u \in C^{1}(\bar{\Omega}): u(z) \geqslant 0 \text { for all } z \in \bar{\Omega},\left.\frac{\partial u}{\partial n}\right|_{\partial \Omega \cap u^{-1}(0)}<0\right\} .
$$

Clearly, this cone has a nonempty interior.

Concerning ordered Banach spaces with an order cone which has a nonempty interior (solid order cone), we have the following result which will be useful in our analysis (see Papageorgiou et al. [15, Proposition 4.1.22]).

Proposition 1 If $X$ is an ordered Banach space with positive (order) cone $K$, int $K$ $\neq \emptyset$, and $e \in$ int $K$, then for every $u \in X$ we can find $\lambda_{u}>0$ such that $\lambda_{u} e-u \in K$.

Let $l \in C^{1}(0, \infty)$ with $l(t)>0$ for all $t>0$. We assume that

$$
\begin{aligned}
& 0<\hat{c} \leqslant \frac{l^{\prime}(t) t}{l(t)} \leqslant c_{0}, c_{1} t^{p-1} \leqslant l(t) \leqslant c_{2}\left[t^{s-1}+t^{p-1}\right] \\
& \text { for all } t>0, \text { and some } c_{1}, c_{2}>0,1 \leqslant s<p .
\end{aligned}
$$

Then the conditions on the map $a(\cdot)$ are the following:

$$
H(a): a(y)=a_{0}(|y|) y \text { for all } y \in \mathbb{R}^{N} \text {, with } a_{0}(t)>0 \text { for all } t>0 \text { and }
$$

(i) $a_{0} \in C^{1}(0,+\infty), t \mapsto a_{0}(t)$ is strictly increasing on $(0,+\infty), a_{0}(t) t \rightarrow 0^{+}$as $t \rightarrow 0^{+}$and

$$
\lim _{t \rightarrow 0^{+}} \frac{a_{0}^{\prime}(t) t}{a_{0}(t)}>-1
$$

(ii) there exists $c_{3}>0$ such that

$$
|\nabla a(y)| \leqslant c_{3} \frac{l(|y|)}{|y|} \text { for all } y \in \mathbb{R}^{N} \backslash\{0\} ;
$$

(iii) $(\nabla a(y) \xi, \xi)_{\mathbb{R}^{N}} \geqslant \frac{l(|y|)}{|y|}|\xi|^{2}$ for all $y \in \mathbb{R}^{n} \backslash\{0\}, \xi \in \mathbb{R}^{N}$;

(iv) if $G_{0}(t)=\int_{0}^{t} a_{0}(s) s d s$, then there exists $\tau \in(q, p]$ such that

$$
\limsup _{t \rightarrow 0^{+}} \frac{\tau G_{0}(t)}{t^{\tau}} \leqslant c^{*}
$$

and $0 \leqslant p G_{0}(t)-a_{0}(t) t^{2}$ for all $t>0$ 
Remark 1 Hypotheses $H(a)(i)$, (ii), ( iii) are dictated by the nonlinear regularity theory of Lieberman [10] and the nonlinear maximum principle of Pucci and Serrin [21]. Hypothesis $H(a)(i v)$ serves the needs of our problem, but in fact, it is a mild condition and it is satisfied in all cases of interest (see the examples below). These conditions were used by Papageorgiou and Rădulescu [13] and by Papageorgiou et al. [16].

Hypotheses $H(a)$ imply that the primitive $G_{0}(\cdot)$ is strictly increasing and strictly convex. We set $G(y)=G_{0}(|y|)$ for all $y \in \mathbb{R}^{N}$. Evidently, $G(\cdot)$ is convex, $G(0)=0$ and

$$
\nabla G(y)=G_{0}^{\prime}(|y|) \frac{y}{|y|}=a_{0}(|y|) y=a(y) \text { for all } y \in \mathbb{R}^{N} \backslash\{0\}, \nabla G(0)=0,
$$

that is, $G(\cdot)$ is the primitive of $a(\cdot)$. From the convexity of $G(\cdot)$ we have

$$
G(y) \leqslant(a(y), y)_{\mathbb{R}^{N}} \text { for all } y \in \mathbb{R}^{N} .
$$

Using hypotheses $H(a)(i)$, (ii), (iii) and (2), we can easily obtain the following lemma, which summarizes the main properties of the map $a(\cdot)$.

Lemma 2 If hypotheses $H(a)(i)$, (ii), (iii) hold, then

(a) the map $y \mapsto a(y)$ is continuous, strictly monotone (hence maximal monotone, too);

(b) $|a(y)| \leqslant c_{4}\left(|y|^{s-1}+|y|^{p-1}\right)$ for some $c_{4}>0$, and all $y \in \mathbb{R}^{N}$;

(c) $(a(y), y)_{\mathbb{R}^{N}} \geqslant \frac{c_{1}}{p-1}|y|^{p}$ for all $y \in \mathbb{R}^{N}$.

Using this lemma and (3), we obtain the following growth estimates for the primitive $G(\cdot)$.

Corollary 3 If hypotheses $H(a)(i)$, (ii), (iii) hold, then $\frac{c_{1}}{p(p-1)}|y|^{p} \leqslant G(y) \leqslant c_{5}(1+$ $\left.|y|^{p}\right)$ for some $c_{5}>0$, and all $y \in \mathbb{R}^{N}$.

The examples that follow confirm that the framework provided by hypotheses $H(a)$ is broad and includes many differential operators of interest (see [13]).

Example 1 (a) $a(y)=|y|^{p-2} y$ with $1<p<\infty$.

This map corresponds to the $p$-Laplace differential operator defined by

$$
\Delta_{p} u=\operatorname{div}\left(|D u|^{p-2} D u\right) \text { for all } u \in W_{0}^{1, p}(\Omega) .
$$

(b) $a(y)=|y|^{p-2} y+\mu|y|^{q-2} y$ with $1<q<p<\infty, \mu \geqslant 0$.

This map corresponds to the $(p, q)$-Laplace differential operator defined by

$$
\Delta_{p} u+\Delta_{q} u \text { for all } u \in W_{0}^{1, p}(\Omega) .
$$

Such operators arise in models of physical processes. We mention the works of Cherfils and Ilyasov [1] (reaction-diffusion systems) and Zhikov [22] (homogenization of composites consisting of two materials with distinct hardening exponent in elasticity theory). 
(c) $a(y)=\left(1+|y|^{2}\right)^{\frac{p-2}{2}} y$ with $1<p<\infty$.

This map corresponds to the modified capillary operator.

(d) $a(y)=|y|^{p-2} y\left(1+\frac{1}{1+|y|^{p}}\right)$ with $1<p<\infty$.

The hypotheses on the potential term $\xi(\cdot)$ and on the singular part $\vartheta(\cdot)$ of the reaction are the following:

$H(\xi): \xi \in L^{\infty}(\Omega)$.

$H(\vartheta): \vartheta:(0,+\infty) \rightarrow(0,+\infty)$ is a locally Lipschitz function such that

(i) for some $\gamma \in(0,1)$ we have

$$
0<c_{6} \leqslant \liminf _{x \rightarrow 0^{+}} \vartheta(x) x^{\gamma} \leqslant \limsup _{x \rightarrow 0^{+}} \vartheta(x) x^{\gamma} \leqslant c_{7}
$$

(ii) $\vartheta(\cdot)$ is nonincreasing.

Remark 2 In the literature we almost always encounter the following particular singular term

$$
\vartheta(x)=x^{-\gamma} \text { for all } x>0 \text {, with } 0<\gamma<1 \text {. }
$$

Of course, hypotheses $H(\vartheta)$ provide a much more general framework and can accomodate also singularities like the ones that follow:

$$
\begin{aligned}
& \vartheta_{1}(x)=x^{-\gamma}[1+\ln (1+x)], \quad x>0, \\
& \vartheta_{2}(x)=x^{-\gamma} e^{-x}, \quad x>0 \\
& \vartheta_{3}(x)=\left\{\begin{array}{ll}
x^{-\gamma}(1-\eta \sin x) & 0<x \leqslant \frac{\pi}{2} \\
x^{-\gamma}(1-\eta) & \text { if } \frac{\pi}{2}<x
\end{array} \text { with } 0<\gamma<1 .\right.
\end{aligned}
$$

The following strong comparison principle can be found in Papageorgiou et al. [16, Proposition 6] (see also Papageorgiou and Smyrlis [17, Proposition 4]).

Proposition 4 If hypotheses $H(a), H(\vartheta)$ hold, $\hat{\xi} \in L^{\infty}(\Omega), \hat{\xi}(z) \geqslant 0$ for almost all $z \in \Omega, h_{1}, h_{2} \in L^{\infty}(\Omega)$ satisfy

$$
0<c_{8} \leqslant h_{2}(z)-h_{1}(z) \text { for almost all } z \in \Omega
$$

and $u, v \in C^{1, \alpha}(\bar{\Omega})$ satisfy $0<u(z) \leqslant v(z)$ for all $z \in \Omega$ and for almost all $z \in \Omega$ we have

- $-\operatorname{div} a(D u(z))-\vartheta(u(z))+\xi(z) u(z)^{p-1}=h_{1}(z)$

- $-\operatorname{div} a\left(D v(z)-\vartheta(v(z))+\xi(z) v(z)^{p-1}=h_{2}(z)\right.$,

then $v-u \in \operatorname{int} \hat{C}_{+}$.

In what follows, $p^{*}$ is the critical Sobolev exponent corresponding to $p$, that is,

$$
p^{*}= \begin{cases}\frac{N p}{N-p} & \text { if } p<N \\ +\infty & \text { if } N \leqslant p\end{cases}
$$


Now we introduce our hypotheses on the nonlinearity $f(z, x)$.

$H(f): f: \Omega \times \mathbb{R} \rightarrow \mathbb{R}_{+}$is a Carathéodory function such that $f(z, 0)=0$ for almost all $z \in \Omega$ and

(i) $f(z, x) \leqslant a(z)\left(1+x^{r-1}\right)$ for almost all $z \in \Omega$, and all $x \geqslant 0$, with $a \in L^{\infty}(\Omega)$, $p<r<p^{*}$

(ii) if $F(z, x)=\int_{0}^{x} f(z, s) d s$, then $\lim _{x \rightarrow+\infty} \frac{F(z, x)}{x^{p}}=+\infty$ uniformly for almost all $z \in \Omega$;

(iii) there exists $\sigma \in\left((r-p) \max \left\{\frac{N}{p}, 1\right\}, p^{*}\right), \sigma>q$ such that

$$
0<\hat{\beta}_{0} \leqslant \liminf _{x \rightarrow+\infty} \frac{f(z, x) x-p F(z, x)}{x^{\sigma}} \text { uniformly for almost all } z \in \Omega
$$

(iv) $\lim \sup _{x \rightarrow 0^{+}} \frac{f(z, x)}{x^{r-1}} \leqslant \eta_{0}$ uniformly for almost all $z \in \Omega$;

(v) for every $\rho>0$, there exists $\hat{\xi}_{\rho}>0$ such that for almost all $z \in \Omega$ the function

$$
x \mapsto f(z, x)+\hat{\xi}_{\rho} x^{\rho-1}
$$

is nondecreasing on $[0, \rho]$.

Remark 3 Since our aim is to find positive solutions and the above hypotheses concern the positive semiaxis $\mathbb{R}_{+}=[0,+\infty)$, we may assume that

$$
f(z, x)=0 \text {, for almost all } z \in \Omega \text {, and all } x \leqslant 0 .
$$

Hypotheses $H(f)(i i)$, (iii) imply that

$$
\lim _{x \rightarrow+\infty} \frac{f(z, x)}{x^{p-1}}=+\infty \text { uniformly for almost all } z \in \Omega .
$$

So, the nonlinearity $f(z, \cdot)$ is $(p-1)$-superlinear near $+\infty$. However, this superlinearity of $f(z, \cdot)$ is not formulated using the AR-condition. We recall that the AR-condition (unilateral version due to (4)), says that there exist $\gamma>p$ and $M>0$ such that

$$
\begin{aligned}
& 0<\gamma F(z, x) \leqslant f(z, x) x \text { for almost all } z \in \Omega, \text { and all } x \geqslant M, \\
& 0<\operatorname{essinf}_{\Omega} F(\cdot, M) .
\end{aligned}
$$

If we integrate (6a) and use (6b), we obtain the weaker condition

$$
\begin{aligned}
& c_{9} x^{\gamma} \leqslant F(z, x) \text { for almost all } z \in \Omega, \text { all } x \geqslant M, \text { and some } c_{9}>0, \\
& \Rightarrow c_{9} x^{\gamma-1} \leqslant f(z, x) \text { for almost all } z \in \Omega, \text { and all } x \geqslant M .
\end{aligned}
$$

Therefore the AR-condition implies that $f(z, \cdot)$ exhibits at least $(\gamma-1)$-polynomial growth. Evidently, (7) implies the much weaker condition (5). In this work instead of the standard AR-condition, we employ the less restrictive hypothesis $H(f)(i i i)$. 
In this way we incorporate in our framework also $(p-1)$-superlinear terms with "slower" growth near $+\infty$, which fail to satisfy the AR-condition. The following function satisfies hypotheses $H(f)$ but fails to satisfy the AR-condition (for the sake of simplicity we drop the $z$-dependence)

$$
f(x)=x^{p-1} \ln (1+x) \text { for all } x \geqslant 0 .
$$

Finally, let us fix the notation which we will use throughout this work. For $x \in \mathbb{R}$ we set $x^{ \pm}=\max \{ \pm x, 0\}$. Then for $u \in W_{0}^{1, p}(\Omega)$ we define $u^{ \pm}(z)=u(z)^{ \pm}$for almost all $z \in \Omega$. It follows that

$$
u^{ \pm} \in W_{0}^{1, p}(\Omega), u=u^{+}-u^{-},|u|=u^{+}+u^{-} .
$$

If $u, v \in W_{0}^{1, p}(\Omega)$ and $u \leqslant v$, then we define.

$$
\begin{aligned}
& {[u, v]=\left\{y \in W_{0}^{1, p}(\Omega): u(z) \leqslant y(z) \leqslant v(z) \text { for almost all } z \in \Omega\right\}} \\
& {[u)=\left\{y \in W_{0}^{1, p}(\Omega): u(z) \leqslant y(z) \text { for almost all } z \in \Omega\right\} .}
\end{aligned}
$$

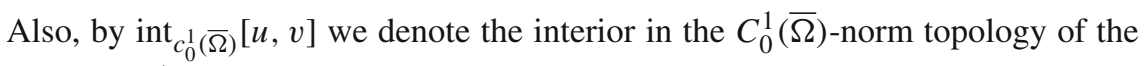
set $[u, v] \cap C_{0}^{1}(\bar{\Omega})$.

By $A: W_{0}^{1, p}(\Omega) \rightarrow W^{-1, p^{\prime}}(\Omega)=W_{0}^{1, p}(\Omega)^{*}\left(\frac{1}{p}+\frac{1}{p^{\prime}}=1\right)$ we denote the nonlinear operator defined by

$$
\langle A(u), h\rangle=\int_{\Omega}(a(D u), D h)_{\mathbb{R}^{N}} d z \text { for all } u, h \in W_{0}^{1, p}(\Omega) .
$$

We know (see Gasinski and Papageorgiou [4]), that $A(\cdot)$ is continuous, strictly monotone (hence maximal monotone, too) and of type $(S)_{+}$, that is,

$$
\begin{aligned}
& \text { "if } u_{n} \stackrel{w}{\rightarrow} u \text { in } W_{0}^{1, p}(\Omega) \text { and } \lim _{\sup _{n \rightarrow \infty}}\left\langle A\left(u_{n}\right), u_{n}-u\right\rangle \leqslant 0, \\
& \text { then } u_{n} \rightarrow u \text { in } W_{0}^{1, p}(\Omega) . "
\end{aligned}
$$

We introduce the following two sets related to problem $\left(P_{\lambda}\right)$ :

$$
\begin{aligned}
& \mathcal{L}=\left\{\lambda>0: \text { problem }\left(P_{\lambda}\right) \text { admits a positive solution }\right\} \\
& S_{\lambda}=\text { the set of positive solutions for problem }\left(P_{\lambda}\right) .
\end{aligned}
$$

We let $\lambda^{*}=\sup \mathcal{L}$.

\section{Positive solutions}

We start by considering the following purely singular problem:

$$
-\operatorname{div} a(D u(z)))+\xi^{+}(z) u(z)^{p-1}=\vartheta(u(z)) \text { in } \Omega,\left.u\right|_{\partial \Omega}=0, u>0 .
$$


From Papageorgiou et al. [16, Proposition 10], we have the following property.

Proposition 5 If hypotheses $H(a), H(\xi), H(\vartheta)$ hold, then problem (8) admits a unique positive solution $v \in$ int $C_{+}$.

Let $\beta>\|\xi\|_{\infty}$. Then hypotheses $H(f)(i)$, (iv) and since $1<q<p<r$, imply that we can find $c_{10}, c_{11}>0$ such that

$\lambda x^{q-1}+f(z, x) \leqslant \lambda c_{10} x^{q-1}+c_{11} x^{r-1}-\beta x^{p-1}$ for almost all $z \in \Omega$, and all $x \geqslant 0$.

Let $k_{\lambda}(x)=\lambda c_{10} x^{q-1}+c_{11} x^{r-1}-\beta x^{p-1}$ for all $x \geqslant 0$. With $v \in \operatorname{int} C_{+}$from Proposition 5, we consider the following auxiliary Dirichlet problem:

$$
\left\{\begin{array}{l}
-\operatorname{div} a(D u(z))+\xi(z) u(z)^{p-1}=\vartheta(v(z))+k_{\lambda}(u(z)) \text { in } \Omega \\
\left.u\right|_{\partial \Omega}=0, u>0 .
\end{array}\right\}
$$

For this problem we prove the following result.

Proposition 6 If hypotheses $H(a), H(\xi), H(\vartheta)$ hold, then for all small enough $\lambda>0$ problem $(10)_{\lambda}$ has a smallest positive solution

$$
\bar{u}_{\lambda} \in \operatorname{int} C_{+} .
$$

Proof Recall that $v \in$ int $C_{+}$(see Proposition 5). Hence $v \in$ int $K_{+}$(see (1)). For $s>N$ we consider the function $\hat{u}_{1}^{1 / s} \in K_{+}$. According to Proposition 1 , we can find $\mu>0$ such that

$$
\begin{aligned}
& \hat{u}_{1}^{1 / s} \leqslant \mu v, \\
& \quad \Rightarrow v^{-\gamma} \leqslant \mu^{\gamma} \hat{u}_{1}^{-\gamma / s} .
\end{aligned}
$$

From the Lemma in Lazer and McKenna [9, p. 726], we have

$$
\begin{aligned}
& \hat{u}_{1}^{-\gamma / s} \in L^{s}(\Omega), \\
& \quad \Rightarrow v^{-\gamma} \in L^{s}(\Omega)(\operatorname{see}(11)) .
\end{aligned}
$$

Hypotheses $H(\vartheta)$ imply that we can find $c_{12}>0$ and $\delta>0$ such that

$$
0 \leqslant \vartheta(x) \leqslant c_{12} x^{-\gamma} \text { for all } 0 \leqslant x \leqslant \delta \text { and } 0 \leqslant \vartheta(x) \leqslant \vartheta(\delta) \text { for all } x>\delta .
$$

It follows from (12), (13) that

$$
\vartheta(v(\cdot)) \in L^{s}(\Omega)(s>N) .
$$

Let $\hat{k}_{\lambda}(x)=\lambda c_{10} x^{q-1}+c_{11} x^{r-1}$ for all $x \geqslant 0$ and set $\hat{K}_{\lambda}(x)=\int_{0}^{x} \hat{k}_{\lambda}(s) d s$. We consider the $C^{1}$-functional $\psi_{\lambda}: W_{0}^{1, p}(\Omega) \rightarrow \mathbb{R}$ defined by

$$
\psi_{\lambda}(u)=\int_{\Omega} G(D u) d z+\frac{1}{p} \int_{\Omega}[\xi(z)+\beta]|u|^{p} d z-\int_{\Omega} \hat{K}_{\lambda}\left(u^{+}\right) d z-\int_{\Omega} \vartheta(v) u^{+} d z
$$


for all $u \in W_{0}^{1, p}(\Omega)$

$\begin{aligned} \geqslant & \frac{c_{1}}{p(p-1)}\|D u\|_{p}^{p}+\frac{1}{p} \int_{\Omega}[\xi(z)+\beta]|u|^{p} d z-\frac{\lambda c_{10}}{q}\|u\|_{q}^{q} \\ & -\frac{c_{11}}{r}\|u\|_{r}^{r}-\int_{\Omega} \vartheta(v)|u| d z\end{aligned}$

(see Corollary 3)

$\geqslant c_{12}\|u\|^{p}-c_{13}\left[\lambda\|u\|+\|u\|^{r}\right]$

for some $c_{12}, c_{13}>0$ and all $0<\lambda \leqslant 1$ (recall that $\beta>\|\xi\|_{\infty}$ and $1<q<r$ )

$=\left[c_{12}-c_{13}\left(\lambda\|u\|^{1-p}+\|u\|^{r-p}\right)\right]\|u\|^{p}$.

We introduce the function $\Im_{\lambda}(t)=\lambda t^{1-p}+t^{r-p}, t>0$. Evidently, $\Im_{\lambda} \in$ $C^{1}(0,+\infty)$ and since $1<p<r$, we see that

$$
\Im_{\lambda}(t) \rightarrow+\infty \text { as } t \rightarrow 0^{+} \text {and as } t \rightarrow+\infty \text {. }
$$

So, we can find $t_{0}>0$ such that

$$
\begin{aligned}
& \Im_{\lambda}\left(t_{0}\right)=\inf \left\{\Im_{\lambda}(t): t>0\right\} \\
& \quad \Rightarrow \Im_{\lambda}^{\prime}\left(t_{0}\right)=0, \\
& \quad \Rightarrow \lambda(p-1) t_{0}^{-p}=(r-p) t_{0}^{r-p-1} \\
& \quad \Rightarrow t_{0}=\left[\frac{\lambda(p-1)}{r-p}\right]^{\frac{1}{r-1}} .
\end{aligned}
$$

Since $\frac{p-1}{r-1}<1$, it follows that

$$
\Im_{\lambda}\left(t_{0}\right) \rightarrow 0 \text { as } \lambda \rightarrow 0^{+} .
$$

So, we can find $\lambda_{0} \in(0,1]$ such that

$$
\Im_{\lambda}\left(t_{0}\right) \leqslant \frac{c_{12}}{c_{13}} \text { for all } \lambda \in\left(0, \lambda_{0}\right] .
$$

For $\rho=t_{0}$, we see from (14) that

$$
\left.\psi_{\lambda}\right|_{\partial \bar{B}_{\rho}}>0
$$

where $\bar{B}_{\rho}=\left\{u \in W_{0}^{1, p}(\Omega):\|u\| \leqslant \rho\right\}$ and $\partial \bar{B}_{\rho}=\left\{u \in W_{0}^{1, p}(\Omega):\|u\|=\rho\right\}$.

We fix $\lambda \in\left(0, \lambda_{0}\right]$. Hypothesis $H(a)(i v)$ implies that we can find $c_{0}^{*}>c^{*}$ and $\delta>0$ such that

$$
G(y) \leqslant \frac{c_{0}^{*}}{\tau}|y|^{\tau} \text { for all }|y| \leqslant \delta
$$

Let $u \in \operatorname{int} C_{+}$and choose small enough $t \in(0,1)$ such that

$$
t|D u(z)| \leqslant \delta \text { for all } z \in \bar{\Omega} \text {. }
$$


Then we have

$$
\psi_{\lambda}(t u) \leqslant \frac{t^{\tau} c_{0}^{*}}{\tau}\|D u\|_{\tau}^{\tau}+\frac{t^{p}}{p} \int_{\Omega}[\xi(z)+\beta]|u|^{p} d z-\frac{\lambda t^{q}}{q}\|u\|_{q}^{q} .
$$

Since $q<\tau \leqslant p$, choosing $t \in(0,1)$ even smaller if necessary, we have

$$
\begin{gathered}
\psi_{\lambda}(t u)<0, \\
\Rightarrow \inf _{\bar{B}_{\rho}} \psi_{\lambda}<0 .
\end{gathered}
$$

The functional $\psi_{\lambda}(\cdot)$ is sequentially weakly lower semicontinuous and by the Eberlein-Smulian theorem and the reflexivity of $W_{0}^{1, p}(\Omega)$, the set $\bar{B}_{\rho}$ is sequentially weakly compact. So, by the Weierstrass-Tonelli theorem, we can find $\bar{u} \in W_{0}^{1, p}(\Omega)$ such that

$$
\psi_{\lambda}(\bar{u})=\inf \left\{\psi_{\lambda}(u): u \in W_{0}^{1, p}(\Omega)\right\} \quad\left(\lambda \in\left(0, \lambda_{0}\right]\right) .
$$

From (15), (16) and (17) it follows that

$$
\begin{aligned}
0 & <\|\bar{u}\|<\rho \\
& \Rightarrow \psi_{\lambda}^{\prime}(\bar{u})=0(\text { see }(17)) \\
& \Rightarrow\langle A(\bar{u}), h\rangle+\int_{\Omega}[\xi(z)+\beta]|\bar{u}|^{p-2} \bar{u} h d z=\int_{\Omega}\left[\vartheta(v)+\hat{k}_{\lambda}\left(\bar{u}^{+}\right)\right] h d z \\
& \text { for all } h \in W_{0}^{1, p}(\Omega)
\end{aligned}
$$

In (18) we choose $h=-\bar{u}^{-} \in W_{0}^{1, p}(\Omega)$. Using Lemma 2(c) and since $\beta>\|\xi\|_{\infty}$ we obtain

$$
\begin{aligned}
& c_{14}\left\|\bar{u}^{-}\right\|^{p} \leqslant 0 \text { for some } c_{14}>0, \\
& \Rightarrow \bar{u} \geqslant 0, \bar{u} \neq 0 .
\end{aligned}
$$

Then from (18) we have

$$
\begin{aligned}
& -\operatorname{div} a(D \bar{u}(z))+\xi(z) \bar{u}(z)^{p-1}=\vartheta(v(z))+k_{\lambda}(\bar{u}(z)) \text { for almost all } z \in \Omega, \\
& \Rightarrow \bar{u} \in W_{0}^{1, p}(\Omega) \text { is a positive of problem }(10)_{\lambda} \text { for } \lambda \in\left(0, \lambda_{0}\right] .
\end{aligned}
$$

From (19) and Theorem 7.1 of Ladyzhenskaya and Uraltseva [8, p. 286], we have $\bar{u} \in L^{\infty}(\Omega)$. Hence $k_{\lambda}(\bar{u}(\cdot)) \in L^{\infty}(\Omega)$. Recall that $\vartheta(v(\cdot)) \in L^{s}(\Omega)$ with $s>N$. From Theorem 9.15 of Gilbarg and Trudinger [6, p. 241], we know that there exists a unique solution $y_{0} \in W^{2, s}(\Omega)$ to the following linear Dirichlet problem

$$
-\Delta y(z)=\vartheta(v(z)) \text { in } \Omega,\left.y\right|_{\partial \Omega}=0 .
$$

By the Sobolev embedding theorem, we have

$$
W^{2, s}(\Omega) \hookrightarrow C^{1, \alpha}(\bar{\Omega}) \text { with } \alpha=1-\frac{N}{s}>0 .
$$


Let $\eta_{0}(z)=D y_{0}(z)$. Then $\eta_{0} \in C^{\alpha}\left(\bar{\Omega}, \mathbb{R}^{N}\right)$ and we have

$$
-\operatorname{div}\left(a(D \bar{u}(z))-\eta_{0}(z)\right)+\xi(z) \bar{u}(z)^{p-1}=k_{\lambda}(\bar{u}(z)) \text { for almost all } z \in \Omega \text {. }
$$

The regularity theory of Lieberman [11] implies that $\bar{u} \in C_{+} \backslash\{0\}$. Moreover, from (19) we have

$$
\begin{aligned}
& \operatorname{div} a(D \bar{u}(z)) \leqslant\|\xi\|_{\infty} \bar{u}(z)^{p-1} \text { for almost all } z \in \Omega \\
& \quad \Rightarrow \bar{u} \in \operatorname{int} C_{+}
\end{aligned}
$$

(from the nonlinear maximum principle, see Pucci and Serrin [21, pp. 111,120]).

Let $\hat{S_{\lambda}}$ denote the set of positive solutions of problem $(10)_{\lambda}$. We have just seen that $\emptyset \neq \hat{S_{\lambda}} \subseteq$ int $C_{+}$for $\lambda \in\left(0, \lambda_{0}\right]$. Moreover, from Papageorgiou et al. [16, Proposition 18], we know that $\hat{S_{\lambda}}$ is downward directed (that is, if $u_{1}, u_{2}, \in \hat{S_{\lambda}}$, then we can find $u \in \hat{S}_{\lambda}$ such that $u \leqslant u_{1}, u \leqslant u_{2}$ ). So, by Lemma 3.10 of Hu and Papageorgiou [7, p. 178], we can find a decreasing sequence $\left\{\bar{u}_{n}\right\}_{n} \geqslant 1 \subseteq \hat{S_{\lambda}}$ such that

$$
\inf \hat{S_{\lambda}}=\inf _{n \geqslant 1} \bar{u}_{n}
$$

For every $n \in \mathbb{N}$ we have

$$
\left\langle A\left(\bar{u}_{n}\right), h\right\rangle+\int_{\Omega} \xi(z) \bar{u}_{n}^{p-1} h d z=\int_{\Omega}\left[\vartheta(v)+k_{\lambda}\left(\bar{u}_{n}\right)\right] h d z \text { for all } h \in W_{0}^{1, p}(\Omega) .
$$

Choosing $h=\bar{u}_{n} \in W_{0}^{1, p}$ and since $0 \leqslant \bar{u}_{n} \leqslant \bar{u}_{1}$ for all $n \in \mathbb{N}$, using Lemma 2(c), we see that $\left\{\bar{u}_{n}\right\}_{n \geqslant 1} \subseteq W_{0}^{1, p}(\Omega)$ is bounded. So, we have

$$
\bar{u}_{n} \stackrel{w}{\rightarrow} \bar{u}_{\lambda} \text { in } W_{0}^{1, p}(\Omega)
$$

Next, in (20) we choose $h=\bar{u}_{n}-\bar{u} \in W_{0}^{1, p}(\Omega)$, pass to the limit as $n \rightarrow \infty$ and use (21). Then

$$
\begin{aligned}
& \lim _{n \rightarrow \infty}\left\langle A\left(\bar{u}_{n}\right), \bar{u}_{n}-\bar{u}_{\lambda}\right\rangle=0 \\
& \quad \Rightarrow \bar{u}_{n} \rightarrow \bar{u}_{\lambda} \text { in } W_{0}^{1, p}(\Omega), \bar{u}_{\lambda} \geqslant 0 \\
& \quad\left(\text { recall that } A(\cdot) \text { is of type }(S)_{+} \text {, see Sect. } 2\right) .
\end{aligned}
$$

We pass to the limit as $n \rightarrow \infty$ in (20) and use (22). Then

$$
\begin{aligned}
& \left\langle A\left(\bar{u}_{\lambda}\right), h\right\rangle+\int_{\Omega} \xi(z) \bar{u}_{\lambda}^{p-1} h d z=\int_{\Omega}\left[\vartheta(v)+k_{\lambda}\left(\bar{u}_{\lambda}\right)\right] h d z \\
& \text { for all } h \in W_{0}^{1, p}(\Omega), \\
& \Rightarrow \bar{u}_{\lambda} \text { is a nonnegative solution of }(10)_{\lambda}
\end{aligned}
$$


Note that for all $n \in \mathbb{N}$, we have

$$
\begin{array}{ll}
-\operatorname{div} a\left(D \bar{u}_{n}(z)\right)+\xi^{+}(z) \bar{u}_{n}(z)^{p-1} & \geqslant \vartheta(v(z))+k_{\lambda}\left(\bar{u}_{n}(z)\right) \\
& \geqslant \vartheta(v(z))=-\operatorname{div} a(D v(z))+\xi^{+}(z) v(z)^{p-1} \\
& \text { for almost all } z \in \Omega, \\
\Rightarrow v \leqslant \bar{u}_{n} \text { for all } n \in \mathbb{N} &
\end{array}
$$

(by the weak comparison principle, see Damascelli [3, Theorem 1.2])

$$
\Rightarrow v \leqslant \bar{u}_{\lambda}(\text { see }(22)) \text {, hence } \bar{u}_{\lambda} \neq 0 \text {. }
$$

Therefore $\bar{u}_{\lambda} \in \hat{S_{\lambda}} \subseteq \operatorname{int} C_{+}$and $\bar{u}_{\lambda}=\inf \hat{S_{\lambda}}$.

We will use $\bar{u}_{\lambda} \in$ int $C_{+}$from Proposition 6 to show the nonemptiness of $\mathcal{L}$.

Proposition 7 If hypotheses $H(a), H(\xi), H(\vartheta), H(f)$ hold, then $\mathcal{L} \neq \varnothing$ and $S_{\lambda} \subseteq$ int $C_{+}$.

Proof From (9) we have

$$
\lambda x^{q-1}+f(z, x) \leqslant k_{\lambda}(x) \text { for almost all } z \in \Omega, \text { and all } x \geqslant 0, \lambda>0 .
$$

For $\lambda \in\left(0, \lambda_{0}\right]$ we have

$$
\begin{aligned}
-\operatorname{div} a\left(D \bar{u}_{\lambda}(z)\right)+\xi(z) \bar{u}_{\lambda}(z)^{p-1}= & \vartheta(v(z))+k_{\lambda}\left(\bar{u}_{\lambda}(z)\right) \\
& (\text { see Proposition 6) } \\
\geqslant & \vartheta\left(\bar{u}_{\lambda}(z)\right)+k_{\lambda}(\bar{u}(z)) \\
& (\text { see }(23) \text { and hypothesis } H(\vartheta)(i i)) \\
\geqslant & \vartheta\left(\bar{u}_{\lambda}(z)\right)+\lambda \bar{u}_{\lambda}(z)^{q-1}+f\left(z, \bar{u}_{\lambda}(z)\right) \\
& \text { for almost all } z \in \Omega(\text { see }(24)) .
\end{aligned}
$$

With $\beta>\|\xi\|_{\infty}$ and $\lambda \in\left(0, \lambda_{0}\right]$, we consider the following truncation-perturbation of the reaction in problem $\left(P_{\lambda}\right)$ :

$$
\gamma_{\lambda}(z, x)= \begin{cases}\vartheta(v(z))+\lambda v(z)^{q-1}+f(z, v(z))+\beta v(z)^{p-1} & \text { if } x<v(z) \\ \vartheta(x)+\lambda x^{q-1}+f(z, x)+\beta x p^{p-1} & \text { if } v(z) \leqslant x \leqslant \bar{u}_{\lambda}(z) \\ \vartheta\left(\bar{u}_{\lambda}(z)\right)+\lambda \bar{u}_{\lambda}(z)^{q-1}+f\left(z, \bar{u}_{\lambda}(z)\right)+\beta \bar{u}_{\lambda}(z)^{p-1} & \text { if } \bar{u}_{\lambda}(z)<x .\end{cases}
$$

This is a Carathéodory function. We set $\Gamma_{\lambda}(z, x)=\int_{0}^{x} \gamma_{\lambda}(z, s) d s$ and consider the functional $\hat{\sigma}_{\lambda}: W_{0}^{1, p}(\Omega) \rightarrow \mathbb{R}$ defined by

$$
\begin{aligned}
& \hat{\sigma}_{\lambda}(u)=\int_{\Omega} G(D u) d z+\frac{1}{p} \int_{\Omega}[\xi(z)+\beta]|u|^{p} d z-\int_{\Omega} \Gamma_{\lambda}(z, u) d z \\
& \text { for all } u \in W_{0}^{1, p}(\Omega) .
\end{aligned}
$$


Using Proposition 3 of Papageorgiou and Smyrlis [17], we see that $\hat{\sigma}_{\lambda} \in$ $C^{1}\left(W_{0}^{1, p}(\Omega)\right)$. Also, from (26), Corollary 3 and since $\beta>\|\xi\|_{\infty}$, we see that $\hat{\sigma}_{\lambda}(\cdot)$ is coercive. In addition, it is sequentially weakly lower semicontinuous. So, we can find $u_{\lambda} \in W_{0}^{1, p}(\Omega)$ such that

$$
\begin{aligned}
& \hat{\sigma}_{\lambda}\left(u_{\lambda}\right)=\inf \left\{\hat{\sigma}(u): u \in W_{0}^{1, p}(\Omega)\right\}, \\
& \quad \Rightarrow \hat{\sigma}_{\lambda}^{\prime}\left(u_{\lambda}\right)=0, \\
& \Rightarrow\left\langle A\left(u_{\lambda}, h\right)\right\rangle+\int_{\Omega}[\xi(z)+\beta]\left|u_{\lambda}\right|^{p-2} u_{\lambda} h d z \\
& =\int_{\Omega} \gamma_{\lambda}\left(z, u_{\lambda}\right) h d z \\
& \quad \text { for all } h \in W_{0}^{1, p}(\Omega) .
\end{aligned}
$$

In (27) first we choose $h=\left(u_{\lambda}-\bar{u}_{\lambda}\right)^{+} \in W_{0}^{1, p}(\Omega)$. Then we have

$$
\begin{aligned}
& \left\langle A\left(u_{\lambda}\right),\left(u_{\lambda}-\bar{u}_{\lambda}\right)^{+}\right\rangle+\int_{\Omega}[\xi(z)+\beta] u_{\lambda}^{p-1}\left(u_{\lambda}-\bar{u}_{\lambda}\right)^{+} d z \\
& =\int_{\Omega}\left[\vartheta\left(\bar{u}_{\lambda}\right)+\lambda \bar{u}_{\lambda}^{q-1}+f\left(z, \bar{u}_{\lambda}\right)+\beta \bar{u}_{\lambda}^{p-1}\right]\left(u_{\lambda}-\bar{u}_{\lambda}\right)^{+} d z(\text { see }(26)) \\
& \leqslant\left\langle A\left(\bar{u}_{\lambda}\right),\left(u_{\lambda}-\bar{u}_{\lambda}\right)^{+}\right\rangle+\int_{\Omega}[\xi(z)+\beta] \bar{u}_{\lambda}^{p-1}\left(u_{\lambda}-\bar{u}_{\lambda}\right)^{+} d z \text { (see (25)), } \\
& \Rightarrow u_{\lambda} \leqslant \bar{u}_{\lambda}\left(\text { since } \beta>\|\xi\|_{\infty}\right) .
\end{aligned}
$$

Next, in (27) we choose $h=\left(v-u_{\lambda}\right)^{+} \in W_{0}^{1, p}(\Omega)$. Then we have

$$
\begin{aligned}
& \left\langle A\left(u_{\lambda}\right),\left(v-u_{\lambda}\right)^{+}\right\rangle+\int_{\Omega}[\xi(z)+\beta]\left|u_{\lambda}\right|^{p-2} u_{\lambda}\left(v-u_{\lambda}\right)^{+} d z \\
& \quad=\int_{\Omega}\left[\vartheta(v)+\lambda v^{q-1}+f(z, v)+\beta v^{p-1}\right]\left(v-u_{\lambda}\right)^{+} d z(\text { see }(26)) \\
& \quad \geqslant \int_{\Omega}\left[\vartheta(v)+\beta v^{p-1}\right]\left(v-u_{\lambda}\right)^{+} d z(\text { since } f \geqslant 0) \\
& =\left\langle A(v),\left(v-u_{\lambda}\right)^{+}\right\rangle+\int_{\Omega}[\xi(z)+\beta] v^{p-1}\left(v-u_{\lambda}\right)^{+} d z \text { (see Proposition 5), } \\
& \Rightarrow v \leqslant u_{\lambda} .
\end{aligned}
$$

So, we have proved that

$$
u_{\lambda} \in\left[v, \bar{u}_{\lambda}\right] \quad\left(\lambda \in\left(0, \lambda_{0}\right]\right) .
$$

It follows from (26), (27) and (28) that

$$
\begin{aligned}
& -\operatorname{div} a\left(D u_{\lambda}(z)\right)+\xi(z) u_{\lambda}(z)^{p-1}=\vartheta\left(u_{\lambda}(z)\right)+\lambda u_{\lambda}(z)^{q-1}+f\left(z, u_{\lambda}(z)\right) \\
& \quad \text { for almost all } z \in \Omega .
\end{aligned}
$$


Note that $\vartheta_{\lambda}\left(u_{\lambda}\right) \leqslant \vartheta(v)$ (see (28) and hypothesis $\left.H(\vartheta)(i i)\right)$ and $\vartheta(v) \in L^{s}(\Omega)$. So, as before (see the proof of Proposition 6), we infer that

$$
u_{\lambda} \in \operatorname{int} C_{+} .
$$

Therefore we have seen that

$$
\left(0, \lambda_{0}\right] \subseteq \mathcal{L}, \text { hence } \mathcal{L} \neq \emptyset
$$

and $S_{\lambda} \subseteq$ int $C_{+}$.

The proof is now complete.

For $\eta>0$, let $\tilde{u}_{\eta} \in$ int $C_{+}$be the unique solution of the following Dirichlet problem

$$
-\operatorname{div} a(D u(z))+\xi^{+}(z) u(z)^{p-1}=\eta \text { in } \Omega,\left.u\right|_{\partial \Omega}=0 .
$$

By Proposition 9 of Papageorgiou et al. [16], we see that given $u \in S_{\lambda} \subseteq$ int $C_{+}$ (that is, $\lambda \in \mathcal{L}$ ), we can find small $\eta>0$ such that

$$
\tilde{u}_{\eta} \leqslant u \text { and } \eta \leqslant \vartheta\left(\tilde{u}_{\eta}\right)
$$

We will use this to obtain a lower bound for the elements of $S_{\lambda}$.

Proposition 8 If hypotheses $H(a), H(\xi), H(\vartheta), H(f)$ hold and $\lambda \in \mathcal{L}$, then $v \leqslant u$ for all $u \in S_{\lambda}$.

Proof Let $u \in S_{\lambda} \subseteq$ int $C_{+}$. Then on account of (29) we can define the following Carathéodory function

$$
e(z, x)= \begin{cases}\vartheta\left(\tilde{u}_{\eta}(z)\right) & \text { if } x<\tilde{u}_{\eta}(z) \\ \vartheta(x) & \text { if } \tilde{u}_{\eta}(z) \leqslant x \leqslant u(z) \\ \vartheta(u(z)) & \text { if } u(z)<x\end{cases}
$$

We set $E(z, x)=\int_{0}^{x} e(z, s) d s$ and consider the functional $\mu: W_{0}^{1, p}(\Omega) \rightarrow \mathbb{R}$ defined by

$$
\mu(u)=\int_{\Omega} G(D u) d z+\frac{1}{p} \int_{\Omega} \xi^{+}(z)|u|^{p} d z-\int_{\Omega} E(z, u) d z \text { for all } u \in W_{0}^{1, p}(\Omega) .
$$

As before, Proposition 3 of Papageorgiou and Smyrlis [17] implies that $\mu \in$ $C^{1}\left(W_{0}^{1, p}(\Omega)\right)$. The coercivity of $\mu(\cdot)$ (see (30)) and the sequential weak lower semicontinuity guarantee the existence of $\tilde{v} \in W_{0}^{1, p}(\Omega)$ such that 


$$
\begin{aligned}
& \mu(\tilde{v})=\inf \left\{\mu(u): u \in W_{0}^{1, p}(\Omega)\right\} \\
& \Rightarrow \mu^{\prime}(\tilde{v})=0, \\
& \Rightarrow\langle A(\tilde{v}), h\rangle+\int_{\Omega} \xi^{+}(z)|\tilde{v}|^{p-2} \tilde{v} h d z=\int_{\Omega} e(z, \tilde{v}) h d z \text { for all } h \in W_{0}^{1, p}(\Omega) .
\end{aligned}
$$

In (31) we choose $h=(\tilde{v}-u)^{+} \in W_{0}^{1, p}(\Omega)$. Then we have

$$
\begin{aligned}
& \left\langle A(\tilde{v}),(\tilde{v}-u)^{+}\right\rangle+\int_{\Omega} \xi^{+}(z) \tilde{v}^{p-1}(\tilde{v}-u)^{+} d z \\
& \quad=\int_{\Omega} \vartheta(u)(\tilde{v}-u)^{+} d z(\operatorname{see}(30)) \\
& \quad \leqslant \int_{\Omega}\left[\vartheta(u)+\lambda u^{q-1}+f(z, u)\right](\tilde{v}-u)^{+} d z\left(\text { since } u \in \operatorname{int} C_{+}, f \geqslant 0\right) \\
& \quad \leqslant\left\langle A(u),(\tilde{v}-u)^{+}\right\rangle+\int_{\Omega} \xi^{+}(z) u^{p-1}(\tilde{v}-u)^{+} d z\left(\text { since } u \in S_{\lambda}\right), \\
& \quad \Rightarrow \tilde{v} \leqslant u .
\end{aligned}
$$

Similarly, if in (31) we choose $h=\left(\tilde{u}_{\eta}-\tilde{v}\right)^{+} \in W_{0}^{1, p}(\Omega)$, then we have

$$
\begin{aligned}
& \left\langle A(\tilde{v}),\left(\tilde{u}_{\eta}-\tilde{v}\right)^{+}\right\rangle+\int_{\Omega} \xi^{+}(z)|\tilde{v}|^{p-2} \tilde{v}\left(\tilde{u}_{\eta}-\tilde{v}\right)^{+} d z \\
& \quad=\int_{\Omega} \vartheta\left(\tilde{u}_{\eta}\right)\left(\tilde{u}_{\eta}-\tilde{v}\right)^{+} d z(\operatorname{see}(30)) \\
& \quad \geqslant \int_{\Omega} \eta\left(\tilde{u}_{\eta}-\tilde{v}\right)^{+} d z(\operatorname{see}(29)) \\
& =\left\langle A\left(\tilde{u}_{\eta}\right),\left(\tilde{u}_{\eta}-v\right)^{+}\right\rangle+\int_{\Omega} \xi^{+}(z) \tilde{u}_{\eta}^{p-1}\left(\tilde{u}_{\eta}-v\right)^{+} d z \\
& \Rightarrow \tilde{u}_{\eta} \leqslant \tilde{v} .
\end{aligned}
$$

So, we have proved that

$$
\tilde{v} \in\left[\tilde{u}_{\eta}, u\right] .
$$

It follows from (30), (31), (32) that $\tilde{v}$ is a positive solution of (18). Then on account of Proposition 5, we have

$$
\begin{aligned}
\tilde{v} & =v \in \operatorname{int} C_{+}, \\
& \Rightarrow v \leqslant u \text { for all } u \in S_{\lambda}(\operatorname{see}(32)) .
\end{aligned}
$$

The proof is now complete.

Next, we show a structural property of the set $\mathcal{L}$, namely that $\mathcal{L}$ is an interval. Moreover, we establish a kind of strong monotonicity property for the solution set $S_{\lambda}$. 
Proposition 9 If hypotheses $H(a), H(\xi), H(\vartheta), H(f)$ hold, $\lambda \in \mathcal{L}, 0<\mu<\lambda$ and $u_{\lambda} \in S_{\lambda} \subseteq$ int $C_{+}$, then $\mu \in \mathcal{L}$ and there exists $u_{\mu} \in S_{\mu} \subseteq \operatorname{int} C_{+}$such that $u_{\lambda}-u_{\mu} \in \operatorname{int} C_{+}$.

Proof From Proposition 8 we know that $v \leqslant u_{\lambda}$. Then with $\beta>\|\xi\|_{\infty}$ we can define the following truncation-perturbation of the reaction in problem $\left(P_{\mu}\right)$ :

$$
e_{\mu}(z, x)= \begin{cases}\vartheta(v(z))+\mu v(z)^{q-1}+f(z, v(z))+\beta v(z)^{p-1} & \text { if } x<v(z) \\ \vartheta(x)+\mu x^{q-1}+f(z, x)+\beta x^{p-1} & \text { if } v(z) \leqslant x \leqslant u_{\lambda}(z) \\ \vartheta\left(u_{\lambda}(z)\right)+\mu u_{\lambda}(z)^{q-1}+f\left(z, u_{\lambda}(z)\right)+\beta u_{\lambda}(z)^{p-1} & \text { if } u_{\lambda}(z)<x\end{cases}
$$

Evidently, $e_{\mu}(z, x)$ is a Carathéodory function. We set $E_{\mu}(z, x)=\int_{0}^{x} e_{\mu}(z, s) d s$ and consider the $C^{1}$-functional $\hat{\psi}_{\mu}: W_{0}^{1, p}(\Omega) \rightarrow \mathbb{R}$ defined by

$$
\begin{aligned}
\hat{\psi}_{\mu}(u)= & \int_{\Omega} G(D u) d z+\frac{1}{p} \int_{\Omega}[\xi(z)+\beta]|u|^{p} d z \\
& -\int_{\Omega} E_{\mu}(z, u) d z \text { for all } u \in W_{0}^{1, p}(\Omega) .
\end{aligned}
$$

Clearly, $\hat{\psi}_{\mu}(\cdot)$ is coercive (see (33) and recall that $\beta>\|\xi\|_{\infty}$ ). It is also sequentially weakly lower semicontinuous. So, we can find $u_{\mu} \in W_{0}^{1, p}(\Omega)$ such that

$$
\begin{aligned}
& \hat{\psi}_{\mu}\left(u_{\mu}\right)=\inf \left\{\hat{\psi}_{\mu}(u): u \in W_{0}^{1, p}(\Omega)\right\} \\
& \quad \Rightarrow \hat{\psi}_{\mu}^{\prime}\left(u_{\mu}\right)=0 \\
& \Rightarrow\left\langle A\left(u_{\mu}\right), h\right\rangle+\int_{\Omega}[\xi(z)+\beta]\left|u_{\mu}\right|^{p-2} u_{\mu} h d z \\
& =\int_{\Omega} e_{\mu}\left(z, u_{\mu}\right) h d z \text { for all } h \in W_{0}^{1, p}(\Omega) .
\end{aligned}
$$

In (34) we first use $h=\left(u_{\mu}-u_{\lambda}\right)^{+} \in W_{0}^{1, p}(\Omega)$. Then

$$
\begin{aligned}
& \left\langle A\left(u_{\mu}\right),\left(u_{\mu}-u_{\lambda}\right)^{+}\right\rangle+\int_{\Omega}[\xi(z)+\beta] u_{\mu}^{p-1}\left(u_{\mu}-u_{\lambda}\right)^{+} d z \\
& \quad=\int_{\Omega}\left[\vartheta\left(u_{\lambda}\right)+\mu u_{\lambda}^{q-1}+f\left(z, u_{\lambda}\right)+\beta u_{\lambda}^{p-1}\right]\left(u_{\mu}-u_{\lambda}\right)^{+} d z \text { (see (33)) } \\
& \leqslant \int_{\Omega}\left[\vartheta\left(u_{\lambda}\right)+\lambda u_{\lambda}^{q-1}+f\left(z, u_{\lambda}\right)+\beta u_{\lambda}^{p-1}\right]\left(u_{\mu}-u_{\lambda}\right)^{+} d z(\text { since } \lambda>\mu) \\
& =\left\langle A\left(u_{\lambda}\right),\left(u_{\mu}-u_{\lambda}\right)^{+}\right\rangle+\int_{\Omega}[\xi(z)+\beta] u_{\lambda}^{p-1}\left(u_{\mu}-u_{\lambda}\right)^{+} d z\left(\text { since } u_{\lambda} \in S_{\lambda}\right), \\
& \Rightarrow u_{\mu} \leqslant u_{\lambda}\left(\text { recall that } \beta>\|\xi\|_{\infty}\right) .
\end{aligned}
$$


Next, in (34) we use $h=\left(v-u_{\mu}\right)^{+} \in W_{0}^{1, p}(\Omega)$. Then from Proposition 5 and since $f \geqslant 0$, we obtain

$$
v \leqslant u_{\mu}
$$

We have proved that

$$
u_{\mu} \in\left[v, u_{\lambda}\right]
$$

It follows from (33), (34), (35) that $u_{\mu} \in S_{\mu} \subseteq$ int $C_{+}$and so $\mu \in \mathcal{L}$.

Let $\rho=\left\|u_{\lambda}\right\|_{\infty}$ and let $\hat{\xi}_{\rho}>0$ as postulated by hypothesis $H(f)(v)$. We have

$$
\begin{aligned}
& -\operatorname{div} a\left(D u_{\mu}\right)+\left[\xi(z)+\hat{\xi}_{\rho}\right] u_{\mu}^{p-1}-\vartheta\left(u_{\mu}\right) \\
& \quad=\mu u_{\mu}^{q-1}+f\left(z, u_{\mu}\right)+\hat{\xi}_{\rho} u_{\mu}^{p-1} \\
& \leqslant \lambda u_{\lambda}^{q-1}+f\left(z, u_{\lambda}\right)+\hat{\xi}_{\rho} u_{\lambda}^{p-1} \text { (see hypothesis } H(f)(v i), \\
& \text { (35) and recall that } \mu<\lambda) \\
& =-\operatorname{div} a\left(D u_{\lambda}\right)+\left[\xi(z)+\hat{\xi}_{\rho}\right] u_{\lambda}^{p-1}-\vartheta\left(u_{\lambda}\right) .
\end{aligned}
$$

From (36) and Proposition 4 of Papageorgiou and Smyrlis [17], we obtain

$$
u_{\lambda}-u_{\mu} \in \operatorname{int} C_{+} .
$$

The proof is now complete.

Proposition 10 If hypotheses $H(a), H(\xi), H(\vartheta), H(f)$ hold, then $\lambda^{*}<+\infty$.

Proof Recall that by hypotheses $H(f)(i i)$, (iii), we have

$$
\lim _{x \rightarrow+\infty} \frac{f(z, x)}{x^{p-1}}=+\infty \text { uniformly for almost all } z \in \Omega
$$

So, we can find $M>0$ such that

$$
f(z, x) \geqslant x^{p-1} \text { for almost all } z \in \Omega \text {, and all } x \geqslant M \text {. }
$$

Hypotheses $H(\vartheta)$ imply that we can find small $\delta \in(0,1]$ such that

$$
\vartheta(x) \geqslant \vartheta(\delta) \geqslant 1 \geqslant \delta^{p-1} \geqslant x^{p-1} \text { for all } x \in(0, \delta] .
$$

Finally, hypotheses $H(f)(i),(v)$ imply that we can find big $\lambda_{0}>0$ such that

$$
\lambda_{0} x^{q-1}+f(z, x) \geqslant x^{p-1} \text { for almost all } z \in \Omega \text { and all } \delta \leqslant x \leqslant M .
$$

Combining (37), (38), (39) we have

$$
\vartheta(x)+\lambda_{0} x^{q-1}+f(z, x) \geqslant x^{p-1} \text { for almost all } z \in \Omega \text { and all } x \geqslant 0 .
$$


Let $\lambda>\lambda_{0}$ and assume that $\lambda \in \mathcal{L}$. Then according to Proposition 7 we can find $u_{\lambda} \in S_{\lambda} \subseteq$ int $C_{+}$. Let $\Omega_{0} \subseteq \Omega$ be an open set with $\bar{\Omega}_{0} \subseteq \Omega$ and $C^{2}$-boundary $\partial \Omega_{0}$. We have

$$
0<m_{0}=\min _{\bar{\Omega}_{0}} u_{\lambda}
$$

For $\epsilon>0$, let $m_{0}^{\epsilon}=m_{0}+\epsilon$ and with $\rho=\left\|u_{\lambda}\right\|_{\infty}$, let $\hat{\xi}_{\rho}>0$ be as postulated by hypothesis $H(f)(v)$. We can always take $\hat{\xi}_{\rho}>\|\xi\|_{\infty}$. We have

$$
\begin{aligned}
- & \operatorname{div} a\left(D m_{0}^{\epsilon}\right)+\left[\xi(z)+\hat{\xi}_{\rho}\right]\left(m_{0}^{\epsilon}\right)^{p-1}-\vartheta\left(m_{0}^{\epsilon}\right) \\
\leqslant & {\left[\xi(z)+\hat{\xi}_{\rho}\right] m_{0}^{p-1}+\chi(\epsilon)-\vartheta\left(m_{0}\right) } \\
& \text { with } \chi(\epsilon) \rightarrow 0^{+} \text {as } \epsilon \rightarrow 0^{+}(\text {see hypotheses } H(\vartheta)) \\
< & {\left[\xi(z)+\hat{\xi}_{\rho}\right] u_{\lambda}^{p-1}+u_{\lambda}^{p-1}-\vartheta\left(u_{\lambda}\right)+\chi(\epsilon) } \\
< & {\left[\xi(z)+\hat{\xi}_{\rho}\right] u_{\lambda}^{p-1}+\lambda_{0} u_{\lambda}^{p-1}+f\left(z, u_{\lambda}\right)+\chi(\epsilon)(\text { see }(40)) } \\
= & {\left[\xi(z)+\hat{\xi}_{\rho}\right] u_{\lambda}^{p-1}+\lambda u_{\lambda}^{q-1}+f\left(z, u_{\lambda}\right)-\left(\lambda-\lambda_{0}\right) u_{\lambda}^{q-1}+\chi(\epsilon) } \\
< & {\left[\xi(z)+\hat{\xi}_{\rho}\right] u_{\lambda}^{p-1}+\lambda u_{\lambda}^{q-1}+f\left(z, u_{\lambda}\right) \text { for } \epsilon>0 \text { small enough } } \\
= & -\operatorname{div} a\left(D u_{\lambda}\right)+\left[\xi(z)+\hat{\xi}_{p}\right] u_{\lambda}^{p-1}-\vartheta\left(u_{\lambda}\right) \\
& \quad \text { for almost all } z \in \Omega_{0}\left(\text { recall that } u_{\lambda} \in S_{\lambda}\right) .
\end{aligned}
$$

Then from (40) and Proposition 4, we see that for small enough $\epsilon>0$ we have

$$
u_{\lambda}-m_{0}^{\epsilon} \in \operatorname{int} \hat{C}_{+}\left(\bar{\Omega}_{0}\right)
$$

which contradicts the definition of $m_{0}$. Hence $\lambda \notin \mathcal{L}$ and so $\lambda^{*} \leqslant \lambda_{0}<+\infty$.

By Propositions 9 and 10 it follows that

$$
\left(0, \lambda^{*}\right) \subseteq \mathcal{L} \subseteq\left(0, \lambda^{*}\right]
$$

Proposition 11 If hypotheses $H(a), H(\xi), H(\vartheta), H(f)$ hold and $\lambda \in\left(0, \lambda^{*}\right)$, then problem $\left(P_{\lambda}\right)$ admits at least two positive solutions

$$
u_{0}, \hat{u} \in \operatorname{int} C_{+}, u_{0} \neq \hat{u} \text {. }
$$

Proof Let $0<\mu<\lambda<\eta<\lambda^{*}$. We have $\mu, \eta \in \mathcal{L}$ (see (42)). On account of Proposition 9 we can find $u_{\mu} \in S_{\mu} \subseteq$ int $C_{+}, u_{0} \in S_{\lambda} \subseteq \operatorname{int} C_{+}, u_{\eta} \in S_{\eta} \subseteq \operatorname{int} C_{+}$ such that

$$
\begin{aligned}
& u_{0}-u_{\eta} \in \operatorname{int} C_{+} \text {and } u_{\eta}-u_{0} \in \operatorname{int} C_{+}, \\
& \Rightarrow u_{0} \in \operatorname{int}_{C_{0}^{1}(\bar{\Omega})}\left[u_{\mu}, u_{\eta}\right] .
\end{aligned}
$$


With $\beta>\|\xi\|_{\infty}$, we introduce the Carathéodory function $d_{\lambda}(z, x)$ defined by

$$
d_{\lambda}(z, x)= \begin{cases}\vartheta\left(u_{\mu}(z)\right)+\lambda u_{\mu}(z)^{q-1}+f\left(z, u_{\mu}(z)\right)+\beta u_{\mu}(z)^{p-1} & \text { if } x \leqslant u_{\mu}(z) \\ \vartheta(x)+\lambda x^{q-1}+f(z, x)+\beta x^{p-1} & \text { if } u_{\mu}(z)<x\end{cases}
$$

We set $D_{\lambda}(z, x)=\int_{0}^{x} d_{\lambda}(z, s) d s$ and consider the functional $\varphi_{\lambda}: W_{0}^{1, p}(\Omega) \rightarrow \mathbb{R}$ defined by

$\varphi_{\lambda}(u)=\int_{\Omega} G(D u) d z+\frac{1}{p} \int_{\Omega}[\xi(z)+\beta]|u|^{p} d z-\int_{\Omega} D_{\lambda}(z, u) d z$ for all $u \in W_{0}^{1, p}(\Omega)$.

We know that $\varphi_{\lambda} \in C^{1}\left(W_{0}^{1, p}(\Omega)\right)$ (see Papageorgiou and Smyrlis [17, Proposition 3]). Also, let

$$
\hat{d}_{\lambda}(z, x)= \begin{cases}d_{\lambda}(z, x) & \text { if } x \leqslant u_{\eta}(z) \\ d_{\lambda}\left(z, u_{\eta}(z)\right) & \text { if } u_{\eta}(z)<x\end{cases}
$$

This is a Carathéodory function. We set $\hat{D}_{\lambda}(z, x)=\int_{0}^{x} \hat{d}_{\lambda}(z, s) d s$ and consider the $C^{1}$-functional $\hat{\varphi}_{\lambda}: W_{0}^{1, p}(\Omega) \rightarrow \mathbb{R}$ defined by

$\hat{\varphi}_{\lambda}(u)=\int_{\Omega} G(D u) d z+\frac{1}{p} \int_{\Omega}[\xi(z)+\beta]|u|^{p} d z-\int_{\Omega} \hat{D}_{\lambda}(z, u) d z$ for all $u \in W_{0}^{1, p}(\Omega)$.

Using (44) and (45) and the nonlinear regularity theory (see the proof of Proposition 7), we show that

$$
\begin{aligned}
& K_{\varphi_{\lambda}} \subseteq\left[u_{\mu}\right) \cap \operatorname{int} C_{+}, \\
& K_{\hat{\varphi}_{\lambda}} \subseteq\left[u_{\mu}, u_{\eta}\right] \cap \operatorname{int} C_{+} .
\end{aligned}
$$

From (47) we see that we can assume that

$$
K_{\hat{\varphi}_{\lambda}}=\left\{u_{0}\right\}
$$

or otherwise we already have a second positive solution for $\left(P_{\lambda}\right)($ see $(45))$ and so we are done.

Clearly, $\hat{\varphi}_{\lambda}(\cdot)$ is coercive (see (45)) and sequentially weakly lower semicontinuous. So, we can find $\hat{u}_{0} \in W_{0}^{1, p}(\Omega)$ such that

$$
\begin{aligned}
& \hat{\varphi}_{\lambda}\left(\hat{u_{0}}\right)=\inf \left\{\hat{\varphi}_{\lambda}(u): u \in W_{0}^{1, p}(\Omega)\right\} \\
& \quad \Rightarrow \hat{u}_{0} \in K_{\hat{\varphi}_{\lambda}} \\
& \Rightarrow \hat{u}_{0}=u_{0}(\operatorname{see}(48)) .
\end{aligned}
$$

But from (44) and (45) we see that

$$
\left.\hat{\varphi}_{\lambda}\right|_{\left[u_{\mu}, u_{\eta}\right]}=\left.\varphi_{\lambda}\right|_{\left[u_{\mu}, u_{\eta}\right]} .
$$


It follows from (43), (49), (50) that

$$
\begin{aligned}
& u_{0} \text { is a local } C_{0}^{1}(\bar{\Omega}) \text {-minimizer of } \varphi_{\lambda}, \\
& \Rightarrow u_{0} \text { is a local } W_{0}^{1, p}(\Omega) \text {-minimizer of } \varphi_{\lambda} \text { (see [5]). }
\end{aligned}
$$

On account of (44) and (46), we may assume that

$$
K_{\varphi_{\lambda}} \text { is finite. }
$$

Otherwise we already have an infinity of positive smooth solutions. From (51), (52) and Theorem 5.7.6 of Papageorgiou et al. [15], we see that we can find small $\rho \in(0,1)$ such that

$$
\varphi_{\lambda}\left(u_{0}\right)<\inf \left\{\varphi_{\lambda}(u):\left\|u-u_{0}\right\|=\rho\right\}=m_{\rho} .
$$

Hypothesis $H(f)(i i)$ and Corollary 3 imply that if $u \in \operatorname{int} C_{+}$, then

$$
\varphi_{\lambda}(t u) \rightarrow-\infty \text { as } t \rightarrow+\infty
$$

Claim $1 \varphi_{\lambda}$ satisfies the C-condition.

Consider a sequence $\left\{u_{n}\right\}_{n} \geqslant 1 \subseteq W_{0}^{1, p}(\Omega)$ such that

$$
\begin{aligned}
& \left|\varphi_{\lambda}\left(u_{n}\right)\right| \leqslant c_{15} \text { for some } c_{15}>0, \text { and all } n \in \mathbb{N}, \\
& \left(1+\left\|u_{n}\right\|\right) \varphi_{\lambda}^{\prime}\left(u_{n}\right) \rightarrow \text { in } W^{-1, p^{\prime}}(\Omega)=W_{0}^{1, p}(\Omega)^{*} \text { as } n \rightarrow \infty .
\end{aligned}
$$

From (56) we have

$$
\begin{aligned}
& \left.\left|\left\langle A\left(u_{n}\right), h\right\rangle+\int_{\Omega}[\xi(z)+\beta]\right| u_{n}\right|^{p-2} u_{n} h d z-\int_{\Omega} d_{\lambda}\left(z, u_{n}\right) h d z \mid \leqslant \frac{\epsilon_{n}\|h\|}{1+\left\|u_{n}\right\|} \\
& \text { for all } h \in W_{0}^{1, p}(\Omega), \text { with } \epsilon_{n} \rightarrow 0^{+} .
\end{aligned}
$$

In (57) we choose $h=-u_{n}^{-} \in W_{0}^{1, p}(\Omega)$. From (44) and Lemma 2, we have

$$
\begin{aligned}
& \frac{c_{1}}{p-1}\left\|D u_{n}^{-}\right\|_{p}^{p}+\int_{\Omega}[\xi(z)+\beta]\left(u_{n}^{-}\right)^{p} d z \leqslant \epsilon_{n}+c_{16}\left\|u_{n}^{-}\right\| \\
& \text {for some } c_{16}>0, \text { and all } n \in \mathbb{N}, \\
& \left.\Rightarrow\left\{u_{n}^{-}\right\}_{n \geqslant 1} \subseteq W_{0}^{1, p}(\Omega) \text { is bounded (recall that } \beta>\|\xi\|_{\infty}\right) .
\end{aligned}
$$


Next, in (57) we choose $h=u_{n}^{+} \in W_{0}^{1, p}(\Omega)$. Then

$$
\begin{aligned}
& -\int_{\Omega}\left(a\left(D u_{n}^{+}\right), D u_{n}^{+}\right)_{\mathbb{R}^{N}} d z-\int_{\Omega}[\xi(z)+\beta]\left(u_{n}^{+}\right)^{p} d z \\
& +\int_{\Omega}\left[\lambda\left(u_{n}^{+}\right)^{q}+f\left(z, u_{n}^{+}\right) u_{n}^{+}\right] d z \leqslant c_{17}
\end{aligned}
$$

for some $c_{17}>0$ and all $n \in \mathbb{N}$ (see (44) and hypothesis $H(\vartheta)(i i)$ ).

From (55) and (58) we obtain

$$
\int_{\Omega} p G\left(D u_{n}^{+}\right) d z+\int_{\Omega}[\xi(z)+\beta]\left(u_{n}^{+}\right)^{p} d z-\int_{\Omega}\left[\frac{\lambda p}{q}\left(u_{n}^{+}\right)^{q}+p F\left(z, u_{n}^{+}\right)\right] d z \leqslant c_{18}
$$

for some $c_{18}>0$ and all $n \in \mathbb{N}$.

Adding (59) and (60) and using hypothesis $H(a)(i v)$, we obtain

$$
\begin{aligned}
& \int_{\Omega}\left[f\left(z, u_{n}^{+}\right) u_{n}^{+}-p F\left(z, u_{n}^{+}\right)\right] d z \leqslant c_{19}+\lambda\left[\frac{p}{q}-1\right]\left\|u_{n}^{+}\right\|_{q}^{q} \\
& \quad \text { for some } c_{19}>0, \text { all } n \in \mathbb{N} .
\end{aligned}
$$

From hypotheses $H(f)($ i $)$, (iii) we see that we can find $\hat{\beta}_{1} \in\left(0, \hat{\beta}_{0}\right)$ and $c_{20}>0$ such that

$$
\hat{\beta}_{1} x^{\sigma}-c_{20} \leqslant f(z, x) x-p F(z, x) \text { for almost all } z \in \Omega \text { and all } x \geqslant 0 \text {. }
$$

Using (62) in (61) and recalling that $q<\sigma$ (see hypothesis $H(f)(i i i)$ ) we obtain that

$$
\left\{u_{n}^{+}\right\}_{n \geqslant 1} \subseteq L^{\sigma}(\Omega) \text { is bounded. }
$$

First, suppose that $N \neq p$. It is clear from hypothesis $H(f)(i i i)$ that we may assume that $\sigma<r<p^{*}$ (recall that $p^{*}=+\infty$ if $N \leqslant p$ ). Let $t \in(0,1)$ be such that

$$
\frac{1}{r}=\frac{1-t}{\sigma}+\frac{t}{p^{*}}
$$

From the interpolation inequality (see, for example, Papageorgiou and Winkert [18, Proposition 2.3.17, p.116]), we have

$$
\begin{aligned}
& \left\|u_{n}^{+}\right\|_{r} \leqslant\left\|u_{n}^{+}\right\|_{\sigma}^{1-t}\left\|u_{n}^{+}\right\|_{p^{*}}^{t}, \\
& \quad \Rightarrow\left\|u_{n}^{+}\right\|_{r}^{r} \leqslant c_{21}\left\|u_{n}^{+}\right\|^{t r} \\
& \text { for some } c_{21}>0 \text { and all } n \in \mathbb{N}(\text { see }(63)) .
\end{aligned}
$$

From hypothesis $H(f)(i)$, we have

$$
f(z, x) x \leqslant c_{22}\left[1+x^{r}\right] \text { for almost all } z \in \Omega \text {, all } x \geqslant 0 \text { and some } c_{22}>0 \text {. }
$$


In (57) we choose $h=u_{n}^{+} \in W_{0}^{1, p}(\Omega)$ and use Lemma 2. Then

$$
\begin{aligned}
& \frac{c_{1}}{p-1}\left\|D u_{n}^{+}\right\|_{p}^{p}+\int_{\Omega}[\xi(z)+\beta]\left(u_{n}^{+}\right)^{p} d z \leqslant \epsilon_{n}+\int_{\Omega} d_{\lambda}\left(z, u_{n}\right) u_{n}^{+} d z, \\
& \Rightarrow \frac{c_{1}}{p-1}\left\|D u_{n}^{+}\right\|_{p}^{p} \leqslant c_{23}+\int_{\Omega}\left[\lambda\left(u_{n}^{+}\right)^{q}+f\left(z, u_{n}^{+}\right) u_{n}^{+}\right] d z \\
& \quad \text { for some } c_{23}>0 \text { and all } n \in \mathbb{N}(\text { see (44)) } \\
& \leqslant c_{24}\left[1+\lambda\left\|u_{n}^{+}\right\|^{q}+\left\|u_{n}^{+}\right\|^{t r}\right] \\
& \quad \text { for some } c_{24}>0 \text { and all } n \in \mathbb{N} \text { (see (64) and (65)). }
\end{aligned}
$$

The hypothesis on $\sigma$ (see $H(f)(i i i)$ ) implies that $t r<p$. Also we have $q<p$. Therefore it follows from (66) that

$$
\left\{u_{n}^{+}\right\}_{n \geqslant 1} \subseteq W_{0}^{1, p}(\Omega) \text { is bounded. }
$$

If $p=N$, then $p^{*}=+\infty$ and by the Sobolev embedding theorem, we have that $W_{0}^{1, p}(\Omega) \hookrightarrow L^{s}(\Omega)$ for all $1 \leqslant s<\infty$. So, we need to replace in the previous argument $p^{*}$ by $s>r>\sigma$ big enough. More precisely, as before, let $t \in(0,1)$ be such that

$$
\begin{aligned}
& \frac{1}{r}=\frac{1-t}{\sigma}+\frac{t}{s}, \\
& \quad \Rightarrow t r=\frac{s(r-\sigma)}{s-\sigma} \rightarrow r-\sigma \text { as } s \rightarrow+\infty
\end{aligned}
$$

Recall that $r-\sigma<p$ (see hypothesis $H(f)($ iii $)$ ). Hence for large enough $s>r$

$$
t r=\frac{s(r-\sigma)}{s-\sigma}<p
$$

Then for such large $s>r$, the previous argument is valid and we again obtain (67). From (58) and (67) we have that $\left\{u_{n}\right\}_{n} \geqslant 1 \subseteq W_{0}^{1, p}(\Omega)$ is bounded. So, we may assume that

$$
u_{n} \stackrel{w}{\rightarrow} u \text { in } W_{0}^{1, p}(\Omega) .
$$

In (57) we choose $h=u_{n}-u \in W_{0}^{1, p}(\Omega)$, pass to the limit as $n \rightarrow \infty$, and use (68). Then

$$
\begin{aligned}
& \lim _{n \rightarrow \infty}\left\langle A\left(u_{n}\right), u_{n}-u\right\rangle=0 \\
& \quad \Rightarrow u_{n} \rightarrow u \text { in } W_{0}^{1, p}(\Omega) \\
& \left.\quad \text { (using the }(S)_{+} \text {property of } A(\cdot), \text { seesee Sect. } 2\right) \\
& \quad \Rightarrow \varphi_{\lambda}(\cdot) \text { satisfies the } C-\text { condition. }
\end{aligned}
$$

This proves Claim 1. 
From (53), (54) and Claim 1, we see that we can apply the mountain pass theorem. So, we can find $\hat{u} \in W_{0}^{1, p}(\Omega)$ such that

$$
\hat{u} \in K_{\varphi_{\lambda}} \subseteq\left[u_{\mu}\right) \cap \operatorname{int} C_{+}\left(\text {see (46)) and } m_{\rho} \leqslant \varphi_{\lambda}(\hat{u})\right. \text { (see (53)). }
$$

It follows rom (44) and (69) that

$$
\hat{u} \in S_{\lambda} \subseteq \text { int } C_{+} \text {and } u_{0} \neq \hat{u} .
$$

The proof is now complete.

Proposition 12 If hypotheses $H(a), H(\xi), H(\vartheta), H(f)$ hold, then $\lambda^{*} \in \mathcal{L}$.

Proof Let $\left\{\lambda_{n}\right\}_{n \geqslant 1} \subseteq\left(0, \lambda^{*}\right)$ be such that $\lambda_{n} \uparrow \lambda^{*}$. We know that $\lambda_{n} \in \mathcal{L}$ for all $n \in \mathbb{N}$ and so we can find $u_{n}=u_{\lambda_{n}} \in S_{\lambda_{n}} \subseteq$ int $C_{+}(n \in \mathbb{N})$ increasing (see Proposition 9).

Let $\hat{\varphi}_{\lambda_{n}}(\cdot)$ be the functional from the proof of Proposition 11, with $u_{\mu}=$ $u_{n-1}, u_{\mu}=u_{n+1}(n \geqslant 2)$. Then we have

$$
\begin{aligned}
\hat{\varphi}_{\lambda_{n}}\left(u_{n}\right) \leqslant & \hat{\varphi}_{\lambda_{n}}\left(u_{n-1}\right) \\
= & \int_{\Omega} G\left(D u_{n-1}\right) d z+\frac{1}{p} \int_{\Omega}[\xi(z)+\beta] u_{n-1}^{p} d z-\int_{\Omega}\left[\vartheta\left(u_{n-1}\right)+\lambda_{n} u_{n-1}^{q-1}\right. \\
& \left.+f\left(z, u_{n-1}+\beta u_{n-1}^{p-1}\right)\right] u_{n-1} d z \\
\leqslant & \int_{\Omega} G\left(D u_{n-1}\right) d z+\frac{1}{p} \int_{\Omega}[\xi(z)+\beta] u_{n-1}^{p} d z-\int_{\Omega}\left[\vartheta\left(u_{n-1}\right)+\lambda_{n-1} u_{n-1}^{q-1}\right. \\
& \left.+f\left(z, u_{n-1}\right)+\beta u_{n-1}^{p-1}\right] u_{n-1} d z \\
\leqslant & \int_{\Omega}\left(a\left(D u_{n-1}\right), D u_{n+1}\right) d z+\int_{\Omega} \xi(z) u_{n-1}^{p} d z-\int_{\Omega}\left[\vartheta\left(u_{n-1}\right)+\lambda_{n-1} u_{n-1}^{q-1}\right. \\
& \left.+f\left(z, u_{n-1}\right)\right] u_{n-1} d z\left(\text { see }(3) \text { and recall that } \beta>\|\xi\|_{\infty}\right) \\
= & 0\left(\text { since } u_{n-1} \in S_{\lambda_{n-1}}\right) .
\end{aligned}
$$

Also, we have

$$
\begin{aligned}
& \left\langle A\left(u_{n}\right), h\right\rangle+\int_{\Omega}[\xi(z)+\beta] u_{n}^{p-1} h d z=\int_{\Omega} d_{\lambda_{n}}\left(z, u_{n}\right) h d z \\
& \text { for all } h \in W_{0}^{1, p}(\Omega) \text { and all } n \in \mathbb{N} .
\end{aligned}
$$

Using (70), (71) and reasoning as in the proof of Proposition 11 (see Claim 1), we obtain that

$$
\left\{u_{n}\right\}_{n \geqslant 1} \subseteq W_{0}^{1, p}(\Omega) \text { is bounded. }
$$

From this, as in the proof of Proposition 11, exploiting the $(S)_{+}$property of $A(\cdot)$, we obtain

$$
u_{n} \rightarrow u_{*} \text { in } W_{0}^{1, p}(\Omega)
$$


Passing to the limit as $n \rightarrow \infty$ in (71) and using (72), we have

$$
u_{*} \in S_{\lambda_{*}} \subseteq \text { int } C_{+} \text {and so } \lambda^{*} \in \mathcal{L}
$$

The proof is now complete.

This proposition implies that

$$
\mathcal{L}=\left(0, \lambda^{*}\right]
$$

Summarizing the situation for problem $\left(P_{\lambda}\right)$, we can state the following bifurcationtype result.

Theorem 13 If hypotheses $H(a), H(\xi), H(\vartheta), H(f)$ hold, then there exists $\lambda^{*}>0$ such that

(a) for all $\lambda \in\left(0, \lambda^{*}\right)$ problem $\left(P_{\lambda}\right)$ has at least two positive solutions

$$
u_{0}, \hat{u} \in \operatorname{int} C_{+}, u_{0} \neq \hat{u}
$$

(b) for $\lambda=\lambda^{*}$ problem $\left(P_{\lambda}\right)$ has at least one positive solution $u_{*} \in$ int $C_{+}$;

(c) for all $\lambda>\lambda^{*}$ problem $\left(P_{\lambda}\right)$ has no positive solutions.

Acknowledgements This research was supported by the Slovenian Research Agency Grants P1-0292, J1-8131, J1-7025, N1-0064, and N1-0083.

\section{Compliance with ethical standards}

Conflict of interest The authors confirm that there is no conflict of interest.

Open Access This article is distributed under the terms of the Creative Commons Attribution 4.0 International License (http://creativecommons.org/licenses/by/4.0/), which permits unrestricted use, distribution, and reproduction in any medium, provided you give appropriate credit to the original author(s) and the source, provide a link to the Creative Commons license, and indicate if changes were made.

\section{References}

1. Cherfils, L., Ilyasov, Y.: On the stationary solution of generalized reaction diffusion equations with $p \& q$ Laplacian. Commun. Pure Appl. Anal. 4, 9-22 (2005)

2. Chu, Y., Gao, R., Sun, Y.: Existence and regularity of solutions to a quasilinear elliptic problem involving variable sources. Bound. Value Probl. 2017, 155 (2017)

3. Damascelli, L.: Comparison theorems for some quasilinear degenerate elliptic operators and applications to symmetry and monotonicity results. Ann. Inst. H. Poincaré-Analyse Non Linéaire 15, 493-516 (1998)

4. Gasinski, L., Papageorgiou, N.S.: Nonlinear Analysis. Chapman \& Hall/CRC, Boca Raton (2006)

5. Giacomoni, J., Schindler, I., Takac, P.: Sobolev versus Hölder local minimizers and existence of multiple solutions for a singular quasilinear equation. Ann. Sc. Norm. Super. Pisa, Ser. V 6, 117-156 (2007)

6. Gilbarg, D., Trundinger, N.S.: Elliptic Partial Differential Equations of Second Order. Springer, Berlin (1998)

7. Hu, S., Papageorgiou, N.S.: Handbook of Multivalued Analysis. Volume I: Theory. Kluwer Academic Publishers, Dordrecht (1997) 
8. Ladyzhenskaya, O., Uraltseva, N.: Linear and Quasilinear Elliptic Equations. Academic Press, New York (1968)

9. Lazer, A., McKenna, P.J.: On a singular nonlinear elliptic boundary value problem. Proc. Am. Math. Soc. 111, 721-730 (1991)

10. Li, Q., Gao, W.: Existence of weak solutions to a class of singular elliptic equations. Mediterr. J. Math. 13, 4917-4927 (2016)

11. Lieberman, G.: The natural generalization of the natural conditions of Ladyzhenskaya and Uraltseva for elliptic equations. Commun. Partial Differ. Equ. 16, 311-361 (1991)

12. Mohammed, A.: Positive solutions of the $p$-Laplacian equation with singular nonlinearity. J. Math. Anal. Appl. 352, 234-245 (2009)

13. Papageorgiou, N.S., Rădulescu, V.D.: Coercive and noncoercive nonlinear Neumann problems with indefinite potential. Forum Math. 28, 545-571 (2016)

14. Papageorgiou, N.S., Rădulescu, V.D., Repovš, D.D.: Positive solutions for nonlinear parametric singular Dirichlet problems. Bull. Math. Sci. 9(2), 1950011 (2019)

15. Papageorgiou, N.S., Rădulescu, V.D., Repovš, D.D.: Nonlinear Analysis-Theory and Methods. Springer, Cham (2019)

16. Papageorgiou, N.S., Rădulescu, V.D., Repovš, D.D.: Nonlinear nonhomogeneous singular problems submitted

17. Papageorgiou, N.S., Smyrlis, G.: A bifurcation-type theorem for singular nonlinear elliptic equations. Methods Appl. Anal. 22, 147-170 (2015)

18. Papageorgiou, N.S., Winkert, P.: Applied Nonlinear Functional Analysis. De Gruyter, Berlin (2018)

19. Papageorgiou, N.S., Winkert, P.: Singular $p$-Laplacian equations with superlinear perturbation. J. Differ. Equ. 266, 1462-1487 (2019)

20. Perera, K., Zhang, Z.: Multiple positive solutions of singular $p$-Laplacian problems by variational methods. Bound. Value Probl. 2005, 3 (2005)

21. Pucci, P., Serrin, J.: The Maximum Principle. Birkhäuser, Basel (2007)

22. Zhikov, V.V.: Averaging of functionals of the calculus of variations and elasticity theory. Math. USSRIzvest. 29, 33-66 (1987)

Publisher's Note Springer Nature remains neutral with regard to jurisdictional claims in published maps and institutional affiliations. 